\title{
Approximate Solution of the Evolution Operator of the Neutrino System
}

\author{
Josefa Surek de Souza de Oliveira ${ }^{1}$, Veríssimo Manoel de Aquino \\ Universidade Estadual de Londrina - Departamento de Física \\ Rodovia Celso Garcia Cid, Pr 445 Km 380 \\ Londrina, Paraná, Brasil, CEP 86051-990. \\ E-mail: josurek@yahoo.com.br, verissimeuel.br
}

Neutrino flavor oscillations have been supposed as an explanation for neutrino discrepancies in varied contexts. One of them is the atmospheric neutrino problem. As a consequence of the spherical geometry of the neutrino source volume, it is predicted that atmospheric neutrino flux is up-down symmetric. If this symmetry is not observed then a possible explanation is neutrino oscillation. In this work the evolution operator of the neutrino system at variable electron density is computed as the product of partial operators in the context of existence of two neutrino flavors with extension for three flavors. The relations between survival probability and energy and mixing angles at vacuum are analysed. The up and down fluxes are calculated considering neutrino oscillation to energy $5 \mathrm{GeV}$ for down neutrinos that cross the upper mantle.

Fifth International Conference on Mathematical Methods in Physics - IC2006 -

Centro Brasileiro de Pesquisas Físicas, Rio de Janeiro, Brazil

24-28 April, 2006

\footnotetext{
$1 \quad$ Speaker
} 


\section{Introduction}

In context of Standard model, neutrinos are considered massless particles. However, observations about solar and atmospheric neutrino fluxes present some results whose possible explanation is the mass attribution to neutrinos, consequently oscillations between flavors can happen $[1,2]$.

Oscillation parameters, vacuum mixing angles and mass-squared differences, are determined through the analysis of neutrino fluxes in the several experiments. In some cases there are limitations in data exploration caused by features as the source extension for solar neutrinos and, in the case of reactor and accelerator neutrinos, the immobility of the source and of the detector. However, these problems can be got round in analysis of atmospheric neutrinos.

Atmospheric neutrinos are produced by the interaction of cosmic rays with the atmosphere and the main production channels are [3]:

$$
\begin{aligned}
\pi^{ \pm} \rightarrow & \mu^{ \pm}+v_{\mu}\left(\bar{v}_{\mu}\right) \\
& \mu^{ \pm} \rightarrow e^{ \pm}+v_{e}\left(\bar{v}_{e}\right)+\bar{v}_{\mu}\left(v_{\mu}\right)
\end{aligned}
$$

It is expected that atmospheric neutrino flux presents up-down symmetry [4]. This symmetry means that the neutrino flux reaching directly a specific point of earth surface with a zenith angles $\theta$ (up-neutrino) is equal to the neutrino flux with an angle $\pi-\theta$ (down-neutrino). If the possibility of neutrino oscillation is admitted, the different paths of up and down neutrinos and the MSW effect modify the situation and up-down asymmetry can take place [3]. Thus, measures of these fluxes can be used to determine the physical parameters that describe neutrino oscillations. In this case, different situations can be explored with the variation of the angle $\theta$ or of the energy of the neutrinos.

In this work the neutrino production and the oscillation probabilities at variable matter density and vacuum are computed so that up and down neutrino fluxes can be analysed considering oscillation possibility. The emphasis is given to the evolution equation of the neutrino system, particularly at variable matter density. In this case the strategy of Lisi is adopted, consisting in to take partial evolution operators [5]. The procedure is applied for a specific situation for which the incidence angle is $70^{\circ}$ and the neutrino energy is $5 \mathrm{GeV}$.

For zenith angle larger than $70^{\circ}$ only the upper mantle is crossed by down neutrinos. Then for the calculations, it is chosen this value for which the largest path difference among up and down neutrinos with a continuous electronic density can be got. The energy of $5 \mathrm{GeV}$ is chosen so that the calculation of the neutrino production of the reference [6] can be used.

\section{Neutrino Production}

Taking atmospheric neutrinos with energy around some $\mathrm{GeV}$ or above the effect of the geomagnetic field is negligible, the showers can be taken as unidimensional and the energy range of the solar neutrino is avoided [4]. For this case, the atmospheric neutrino production can be obtained by analytical methods [6].

Following the procedure adopted by Lipari [6], the neutrino production is obtained starting from the meson and muon fluxes of the atmospheric showers; these are related to the primary 
flux whose composition can be approximate as being of nucleons. The meson and muon fluxes are calculated in the high and low energy limits and the generalization is made by interpolation.

Besides, the hadronic interaction lengths are considered constant, the fluxes are described as a power law in the energy, it is admitted that hadrons are not produced due to decays and that they don't lose considerable energy. The polarization [7, 8] and energy loss [9] of the muons are taken into account. The parameters that describe the primary flux, the interaction lengths, the hadronic interaction factors and the muon energy loss parameters are supplied by Gaisser [10]. The model of the atmosphere necessary to analyse the interaction of cosmic rays is described by [6]. The muon neutrino production rate as a function of the distance between the source and a detector for energy of $5 \mathrm{GeV}$ and zenith angle of $70^{\circ}$ is obtained and it is represented graphically in the figure 1 .

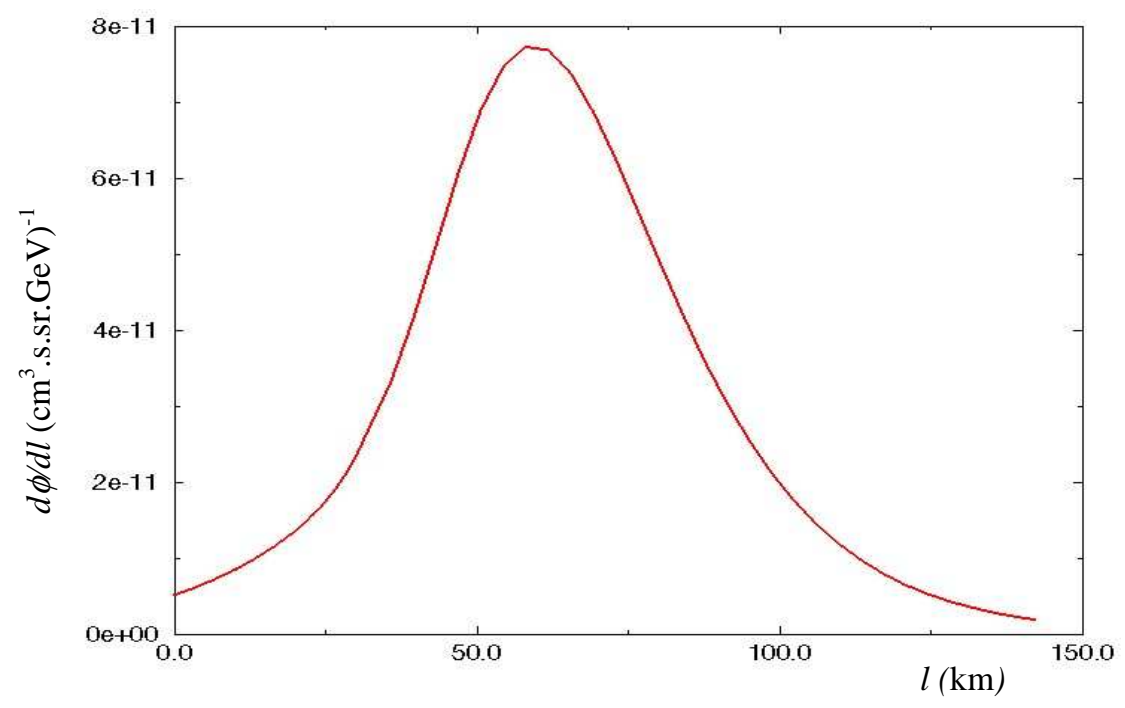

Figure 1: Muon neutrino production rate as a function of the distance between the source and an eventual detector.

\section{Oscillations of Neutrinos}

Attributing Dirac or Majorana mass to neutrinos, their propagation in the ultra-relativistic limit is described by the Schrödinger equation [11] that in the base of flavor states $\mathbf{v}_{f}$ to neutrinos of energy $\mathrm{E}$ is given by

$$
i \frac{\mathrm{d}}{d x} \mathbf{v}_{f}(x)=\frac{1}{2 E}\left(\mathbf{U M}^{2} \mathbf{U}^{-1}+\mathbf{A}\right) \mathbf{v}_{f}(x)
$$

where $\mathbf{U}$ couples flavor and mass eigenstates as $\mathbf{v}_{f}(x)=\mathbf{U} \mathbf{v}_{p}(x)$ and $\mathbf{M}$ is diagonal matrix of mass of the neutrinos. In the context of three neutrino flavors, $\mathbf{U}=e^{i \psi \Lambda_{7}} e^{i \phi \Lambda_{5}} e^{i \omega \Lambda_{2}}$, where $\Lambda_{2}, \Lambda_{5}$ e $\Lambda_{7}$ are Gell-Mann matrices. The diagonal matrix of squared-mass, the flavor and mass eigenvectors are defined in this case respectively by the relations:

$$
\mathrm{M}^{2}=\left(\begin{array}{ccc}
m_{1}^{2} & 0 & 0 \\
0 & m_{2}^{2} & 0 \\
0 & 0 & m_{3}^{2}
\end{array}\right), \quad v_{f}(x)=\left(\begin{array}{c}
v_{e}(x) \\
v_{\mu}(x) \\
v_{\tau}(x)
\end{array}\right) \quad \text { and } \quad v_{p}(x)=\left(\begin{array}{c}
v_{1}(x) \\
v_{2}(x) \\
v_{3}(x)
\end{array}\right) \text {, }
$$


where the states $v_{1,2,3}$ represent particles of masses $m_{1,2,3}$.

The term A contains the matter effect on the neutrino propagation that, in this case, is

$$
\mathbf{A}=\left(\begin{array}{lll}
A & 0 & 0 \\
0 & 0 & 0 \\
0 & 0 & 0
\end{array}\right)
$$

with $A=2 \sqrt{2} G_{F} N_{e} E$, for which $G_{F}$ is the Fermi Constant and $N_{e}$ the electronic density in matter [12]. In the mass base, the equation (1) becomes

$$
i \frac{\mathrm{d}}{d x} \mathbf{v}_{p}(x)=\frac{1}{2 E}\left(\mathbf{M}^{2}+\mathbf{U}^{-1} \mathbf{A} \mathbf{U}\right) \mathbf{v}_{p}(x) .
$$

The solution of (1) in a general way is $\mathbf{v}_{f}(x)=\mathbf{G}\left(x, x_{0}\right) \mathbf{v}_{f}\left(x_{0}\right)$, where $\mathbf{G}\left(x, x_{0}\right)$ is the evolution operator of the neutrino system. Or in terms of particle eigenstates (2), $\mathbf{v}_{p}(x)=\overline{\mathbf{G}}\left(x, x_{0}\right) \mathbf{v}_{p}\left(x_{0}\right)$, where $\overline{\mathbf{G}}\left(x, x_{0}\right)=\mathbf{U}^{-1} \mathbf{G}\left(x, x_{0}\right) \mathbf{U}$. The evolution operator is explicitly:

$$
\mathbf{G}\left(x, x_{0}\right)=\operatorname{Exp}\left[-i \int_{x_{0}}^{x} \mathbf{H}\left(x^{\prime}\right) \mathrm{d} x^{\prime}\right]
$$

where $\mathbf{H}(x)=\frac{1}{2 E}\left(\mathbf{U M}^{2} \mathbf{U}^{-1}+\mathbf{A}\right)$ is the Hamiltonian of the system and the symbol Exp concern to expansionals that represent a expansion in orderly multiple integrals.

$$
\operatorname{Exp}\left[-i \int_{0}^{x} \mathbf{H}\left(x^{\prime}\right) \mathrm{d} x^{\prime}\right]=1-i \int_{0}^{x} \mathbf{H}\left(x^{\prime}\right) \mathrm{d} x^{\prime}+(-i)^{2} \int_{0}^{x} \mathbf{H}\left(x^{\prime}\right) \mathrm{d} x^{\prime} \int_{0}^{x^{\prime}} \mathbf{H}\left(x^{\prime \prime}\right) \mathrm{d} x^{\prime \prime}+\ldots
$$

that for $\left[\mathbf{H}(x), \mathbf{H}\left(x^{\prime}\right)\right]=0$ it is reduced to usual exponential. This is the case of neutrinos traveling the vacuum and the matter of constant density, because in these two situations the Hamiltonian is independent of the neutrino position.

At vacuum the neutrino evolution equation as a function of $\mathbf{v}_{p}$ is reduced to the equation

$$
i \frac{\mathrm{d}}{d x} \mathbf{v}_{p}(x)=\frac{1}{2 E} \mathbf{M}^{2} \mathbf{v}_{p}(x),
$$

whose solution in mass base is easily obtained for $j=1,2,3$ as

$$
v_{j}(x)=e^{-i \frac{m_{j}^{2}}{2 E}\left(x-x_{0}\right)} v_{j}\left(x_{0}\right) \text {. }
$$

The probabilities of $v_{\mu}$, for example, to be detected as $v_{e}, v_{\mu}$ or $v_{\tau}$ after traveling a distance $x$ are respectively

$$
\begin{gathered}
P\left(v_{\mu}, v_{\mu}\right)=1-4 U_{22}^{2} U_{21}^{2} \sin ^{2}\left(\frac{\Delta_{21}}{4 E} x\right)-4 U_{23}^{2} U_{21}^{2} \sin ^{2}\left(\frac{\Delta_{31}}{4 E} x\right)-4 U_{23}^{2} U_{22}^{2} \sin ^{2}\left(\frac{\Delta_{32}}{4 E} x\right), \\
P\left(v_{\mu}, v_{e}\right)=-4 U_{11} U_{12} U_{21} U_{22} \sin ^{2}\left(\frac{\Delta_{21}}{4 E} x\right)-4 U_{13} U_{23}\left[U_{11} U_{21} \sin ^{2}\left(\frac{\Delta_{31}}{4 E} x\right)+U_{12} U_{22} \sin ^{2}\left(\frac{\Delta_{32}}{4 E} x\right)\right] \\
P\left(v_{\mu}, v_{\tau}\right)=-4 U_{32} U_{22} U_{31} U_{21} \sin ^{2}\left(\frac{\Delta_{21}}{4 E} x\right)-4 U_{33} U_{23}\left[U_{31} U_{21} \sin ^{2}\left(\frac{\Delta_{31}}{4 E} x\right)+U_{32} U_{22} \sin ^{2}\left(\frac{\Delta_{32}}{4 E} x\right)\right],
\end{gathered}
$$

where $U_{i j}$ are elements of the unitary matrix $\mathbf{U}$. 
In the case of neutrinos that cross matter of constant density, the Hamiltonian is diagonalized for the unitary matrix $\tilde{\mathbf{U}}$ and (1) becomes

$$
i \frac{\mathrm{d}}{d x} \tilde{\mathbf{v}}_{p}(x)=\frac{1}{2 E} \tilde{\mathbf{M}}^{2} \tilde{\mathbf{v}}_{p}(x),
$$

where effective states $\tilde{\mathbf{v}}_{p}(x)$ are coupled to flavor states as $\mathbf{v}_{f}(x)=\tilde{\mathbf{U}} \tilde{\mathbf{v}}_{p}(x)$ and the diagonal matrix of effective squared-mass is $\tilde{\mathbf{M}}^{2}=\tilde{\mathbf{U}}^{-1}\left(\mathbf{U M}^{2} \mathbf{U}^{-1}+\mathbf{A}\right) \tilde{\mathbf{U}}$.

In terms of $\tilde{\mathbf{v}}_{p}(x)$ the solution of evolution equation to constant matter density is

$$
\tilde{v}_{j}(x)=e^{-i \frac{\tilde{m}_{j}^{2}}{2 E}\left(x-x_{0}\right)} \tilde{v}_{j}\left(x_{0}\right) \text {. }
$$

Therefore, the survival and transition probabilities amplitudes for muon neutrinos are

$$
\begin{aligned}
& P\left(v_{\mu}, v_{\mu}\right)=1-4 \tilde{U}_{22}^{2} \tilde{U}_{21}^{2} \sin ^{2}\left(\frac{\tilde{\Delta}_{21}}{4 E} x\right)-4 \tilde{U}_{23}^{2} \tilde{U}_{21}^{2} \sin ^{2}\left(\frac{\tilde{\Delta}_{31}}{4 E} x\right)-4 \tilde{U}_{23}^{2} \tilde{U}_{22}^{2} \sin ^{2}\left(\frac{\tilde{\Delta}_{32}}{4 E} x\right), \\
& P\left(v_{\mu}, v_{e}\right)=-4 \tilde{U}_{11} \tilde{U}_{12} \tilde{U}_{21} \tilde{U}_{22} \sin ^{2}\left(\frac{\tilde{\Delta}_{21}}{4 E} x\right)-4 \tilde{U}_{13} \tilde{U}_{23}\left[\tilde{U}_{11} \tilde{U}_{21} \sin ^{2}\left(\frac{\tilde{\Delta}_{31}}{4 E} x\right)+\tilde{U}_{12} \tilde{U}_{22} \sin ^{2}\left(\frac{\tilde{\Delta}_{32}}{4 E} x\right)\right] \\
& P\left(v_{\mu}, v_{\tau}\right)=-4 \tilde{U}_{32} \tilde{U}_{22} \tilde{U}_{31} \tilde{U}_{21} \sin ^{2}\left(\frac{\tilde{\Delta}_{21}}{4 E} x\right)-4 \tilde{U}_{33} \tilde{U}_{23}\left[\tilde{U}_{31} \tilde{U}_{21} \sin ^{2}\left(\frac{\tilde{\Delta}_{31}}{4 E} x\right)+\tilde{U}_{32} \tilde{U}_{22} \sin ^{2}\left(\frac{\tilde{\Delta}_{32}}{4 E} x\right)\right] \text {, }
\end{aligned}
$$

here $\tilde{U}_{i j}$ represents the elements of matrix $\tilde{\mathbf{U}}$ and $\tilde{\Delta}_{i j}=\tilde{m}_{i}^{2}-\tilde{m}_{j}^{2}$. Considering the typical energies and paths of atmospheric neutrinos $[13,14]$ and $\tilde{\Delta}_{31} \approx \tilde{\Delta}_{32} \gg \tilde{\Delta}_{21}$,

$$
\begin{gathered}
P\left(v_{\mu}, v_{\mu}\right) \simeq 1-4 \tilde{U}_{23}^{2}\left(\tilde{U}_{21}^{2}+\tilde{U}_{22}^{2}\right) \sin \left(\frac{\tilde{\Delta}_{31}^{2}}{4 E} x\right) \simeq 1-4 \sin ^{2} \tilde{\psi} \cos ^{2} \tilde{\phi}\left(1-\sin ^{2} \tilde{\psi} \cos ^{2} \tilde{\phi}\right) \sin \left(\frac{\tilde{\Delta}_{31}^{2}}{4 E} x\right), \\
P\left(v_{\mu}, v_{x}\right) \simeq 4\left(\tilde{U}_{13}^{2}+\tilde{U}_{33}^{2}\right) \tilde{U}_{23}^{2} \sin \left(\frac{\tilde{\Delta}_{31}^{2}}{4 E} x\right) \simeq 4 \sin ^{2} \tilde{\psi} \cos ^{2} \tilde{\phi}\left(1-\sin ^{2} \tilde{\psi} \cos ^{2} \tilde{\phi}\right) \sin \left(\frac{\tilde{\Delta}_{31}^{2}}{4 E} x\right),
\end{gathered}
$$

with $P\left(v_{\mu}, v_{x}\right)=P\left(v_{\mu}, v_{e}\right)+P\left(v_{\mu}, v_{\tau}\right)$. Moreover it is gotten in ref. [11] that $\tilde{\psi} \rightarrow \psi$ and equations to $\sin ^{2} 2 \tilde{\phi}$ and $\tilde{\Delta}_{31}$ recover that ones obtained in the context of existence of two flavors approach:

$$
\begin{aligned}
\sin ^{2} 2 \tilde{\phi} & \simeq \frac{\Delta_{31}^{2} \sin ^{2} 2 \phi}{\left(\Delta_{31} \cos 2 \phi-A\right)^{2}+\Delta_{31}^{2} \sin ^{2} 2 \phi} \\
\tilde{\Delta}_{31} & \simeq \sqrt{\Delta_{31}^{2}+A^{2}-2 A \Delta_{31} \cos 2 \phi} .
\end{aligned}
$$

Hence, from the definition

$$
\sin ^{2} 2 \tilde{\alpha} \equiv 4 \sin ^{2} \psi \cos ^{2} \tilde{\phi}\left(1-\sin ^{2} \psi \cos ^{2} \tilde{\phi}\right),
$$

follows that the survival and transition probabilities of muon neutrinos can be rewritten as

$$
\begin{gathered}
P\left(v_{\mu}, v_{\mu}\right) \simeq 1-\sin ^{2} 2 \tilde{\alpha} \sin ^{2} \frac{\tilde{\Delta}_{31} t}{4 E} \\
P\left(v_{\mu}, v_{x}\right) \simeq \sin ^{2} 2 \tilde{\alpha} \sin ^{2} \frac{\tilde{\Delta}_{31} t}{4 E}
\end{gathered}
$$

that are similar to those obtained in the two flavor context. The difference is the mixing angle redefined as $\tilde{\alpha}$. 
For neutrinos propagating in an inhomogeneous medium, that is the case of that cross the Earth, the Hamiltonian term that takes into account the interaction with the matter depends on their position. Thus, $\left[H(x), H\left(x^{\prime}\right)\right] \neq 0$ and the evolution operator must be written as a function of expansion in eq. (3), making difficult the treatment of the evolution operator.

The strategy of Lisi [5] is to divide the path in partial elements $j$ in which the electronic density is given by $N_{j}(x)$ and the neutrino propagation is described by the Hamiltonian $\mathbf{H}_{j}(x)$. The evolution operator is taken as a partial operator product

$$
\mathbf{G}\left(x_{F}, x_{0}\right)=\prod_{j} \mathbf{G}_{j}\left(x_{j}, x_{j-1}\right) .
$$

In the reference [5], the simplified situation of oscillation between two flavors is considered, for which the equation (1) assumes the explicit form

$$
i \frac{\mathrm{d}}{\mathrm{d} x} \mathbf{v}_{f}(x)=\frac{1}{4 E}\left[\left(\sum_{21}+A\right)+\left(\begin{array}{cc}
A(x)-\Delta_{21} \cos 2 \theta & \Delta_{21} \sin 2 \theta \\
\Delta_{21} \sin 2 \theta & \Delta_{21} \cos 2 \theta-A(x)
\end{array}\right)\right] \mathbf{v}_{f}(x)
$$

here $\Delta_{21}=m_{2}^{2}-m_{1}^{2}, \sum_{21}=m_{2}^{2}+m_{1}^{2}, A=2 \sqrt{2} G_{F} E N_{e}(x)$ and $\theta$ is the mixing angle.

Thus, the Hamiltonian, except for the proportional term to the identity $\left(\sum_{21}+A\right)$ that just introduces in the evolution operator a constant phase factor, can be defined in each partial element, with $k=\frac{\Delta_{21}}{2 E}$, as

$$
\mathbf{H}_{j}=\frac{1}{2}\left(\begin{array}{cc}
\sqrt{2} G_{F} N_{j}(x)-k \cos 2 \theta & k \sin 2 \theta \\
k \sin 2 \theta & k \cos 2 \theta-\sqrt{2} G_{F} N_{j}(x)
\end{array}\right) .
$$

The partial evolution operator $\mathbf{G}_{j}\left(x_{j}, x_{j-1}\right)$ is associated to this Hamiltonian $\mathbf{H}_{j}$.

The electronic density in the path element is defined as the average electronic density plus a perturbation term $N_{j}(x)=\bar{N}_{j}+\delta N_{j}(x)$, so $\mathbf{H}_{j}=\overline{\mathbf{H}}_{j}+\delta \mathbf{H}_{j}(x)$. The constant part of $\mathbf{H}_{j}$ is

$$
\overline{\mathbf{H}}_{j}=\frac{1}{2}\left(\begin{array}{cc}
\sqrt{2} G_{F} \bar{N}_{j}-k \cos 2 \theta & k \sin 2 \theta \\
k \sin 2 \theta & k \cos 2 \theta-\sqrt{2} G_{F} \bar{N}_{j}
\end{array}\right)
$$

and the perturbation

$$
\delta \mathbf{H}_{j}(x)=\frac{\sqrt{2}}{2} G_{F}\left(\begin{array}{cc}
\delta \bar{N}_{j}(x) & 0 \\
0 & -\delta \bar{N}_{j}(x)
\end{array}\right) .
$$

Then,

$$
\mathbf{v}_{f}\left(x_{F}\right)=\prod_{j} \mathbf{U}_{j}\left(x_{j}, x_{j-1}\right) \mathbf{v}_{f}\left(x_{I}\right)
$$

where the partial evolution operator is

$$
\mathbf{U}_{j}\left(x_{j}, x_{j-1}\right)=\operatorname{Exp}\left\{-i \int_{x_{j-1}}^{x_{j}}\left[\overline{\mathbf{H}}_{j}+\delta \mathbf{H}_{j}(x)\right] \mathrm{d} x\right\} .
$$

As $\overline{\mathbf{H}}_{j}$ is constant,

$$
\mathbf{U}_{j}\left(x_{j}, x_{j-1}\right)=e^{-i\left(x_{j}-x_{j-1}\right) \overline{\mathbf{H}}_{j}} \operatorname{Exp}\left[-i \int_{x_{j-1}}^{x_{j}} \mathrm{~d} x e^{-i\left(x_{j-1}-x\right) \overline{\mathbf{H}}_{j}} \delta \mathbf{H}_{j}(x) e^{-i\left(x-x_{j-1}\right) \overline{\mathbf{H}}_{j}}\right] .
$$


Dividing the path in small elements, the partial evolution operator, in first approximation order, can be written as

$$
\mathbf{U}_{j}\left(x_{j}, x_{j-1}\right) \simeq e^{-i\left(x_{j}-x_{j-1}\right) \overline{\mathbf{H}}_{j}}-i \int_{x_{j-1}}^{x_{j}} \mathrm{~d} x e^{-i\left(x_{j-1}-x\right) \overline{\mathbf{H}}_{j}} \delta \mathbf{H}_{j}(x) e^{-i\left(x-x_{j-1}\right) \overline{\mathbf{H}}_{j}} .
$$

Performing the integral in Eq. (17), the following expression is obtained

$$
\begin{aligned}
\mathbf{U}_{j}\left(x_{j}, x_{j-1}\right) & =\left(\begin{array}{cc}
c_{j}+i s_{j} \cos 2 \tilde{\theta} & -i s_{j} \sin 2 \tilde{\theta} \\
-i s_{j} \sin 2 \tilde{\theta} & c_{j}-i s_{j} \cos 2 \tilde{\theta}
\end{array}\right) \\
& -\frac{i}{2} \sin 2 \tilde{\theta}\left(\begin{array}{cc}
\sin 2 \tilde{\theta} C_{j} & \cos 2 \tilde{\theta} C_{j}-i \sin 2 \tilde{\theta} S_{j} \\
\cos 2 \tilde{\theta} C_{j}+i \sin 2 \tilde{\theta} S_{j} & -\sin 2 \tilde{\theta} C_{j}
\end{array}\right),
\end{aligned}
$$

where

$$
\begin{aligned}
c_{j}=\cos \left(k_{m} \frac{x_{j}-x_{j-1}}{2}\right), & s_{j}=\sin \left(k_{m} \frac{x_{j}-x_{j-1}}{2}\right), \\
C_{j}=\sqrt{2} G_{F} \int_{x_{j-1}}^{x_{j}} \mathrm{~d} x \delta N_{j}(x) \cos \left(k_{m}(x-\bar{x})\right), & S_{j}=\sqrt{2} G_{F} \int_{x_{j-1}}^{x_{j}} \mathrm{~d} x \delta N_{j}(x) \sin \left(k_{m}(x-\bar{x})\right)
\end{aligned}
$$

and $\bar{x}=\frac{x_{j}+x_{j-1}}{2}, \tilde{\Delta}_{21}=\sqrt{\Delta_{21}^{2}+\bar{A}^{2}-2 \bar{A} \Delta_{21} \cos 2 \theta}, \sin 2 \tilde{\theta}=\frac{\Delta_{21}}{\tilde{\Delta}_{21}} \sin 2 \theta, k_{m}=\frac{\tilde{\Delta}_{21}}{2 E}$.

However, the partial evolution operator the evolution operator in first order approximation as presented in Eq. (18) it is not automatically unitary, being necessary to normalize it. The unitarity of $\mathbf{U}_{j}\left(x_{j}, x_{j-1}\right)$ is guaranteed by the redefinition

$$
\mathbf{U}_{j}^{\prime}\left(x_{j}, x_{j-1}\right)=\frac{2}{\sqrt{4+\sin ^{2} 2 \tilde{\theta}\left(S_{j}^{2}+C_{j}^{2}\right)}} \mathbf{U}_{j}\left(x_{j}, x_{j-1}\right)
$$

As discussed previously, for atmospheric neutrinos the oscillation probabilities among three flavors can be described as a function of the oscillation probabilities among two flavors, being enough to redefine the mixture angle $\tilde{\alpha}$ giving in Eq. (8). Thus the survival and transition probabilities of muon neutrinos at variable matter density in the context of three flavors can be defined as oscillation probabilities between muon and another type of neutrino obtained from the partial evolution operator with effective mixing angle $\tilde{\alpha}$

$$
\begin{array}{r}
\mathbf{U}_{j}^{\prime}\left(x_{j}, x_{j-1}\right)=\frac{2}{\sqrt{4+\sin ^{2} 2 \tilde{\alpha}\left(S_{j}^{2}-C_{j}^{2}\right)}}\left(\begin{array}{cc}
c_{j}+i s_{j} \cos 2 \tilde{\alpha} & -i s_{j} \sin 2 \tilde{\alpha} \\
-i s_{j} \sin 2 \tilde{\alpha} & c_{j}-i s_{j} \cos 2 \tilde{\alpha}
\end{array}\right) \\
-\frac{i}{2} \sin 2 \tilde{\alpha}\left(\begin{array}{cc}
\sin 2 \tilde{\alpha} C_{j} & \cos 2 \tilde{\alpha} C_{j}-i \sin 2 \tilde{\alpha} S_{j} \\
\cos 2 \tilde{\alpha} C_{j}+i \sin 2 \tilde{\alpha} S_{j} & -\sin 2 \tilde{\alpha} C_{j}
\end{array}\right)
\end{array}
$$

Treating of atmospheric neutrinos, it is used the Earth electronic density described by PREM [15]. The vacuum oscillation parameters used in the calculations are $\Delta_{32}=3 \times 10^{-3} \mathrm{eV}^{2}$, $\sin ^{2} \psi=0,6$ and $\sin ^{2} \phi=0,01$ supplied by the reference [13]. The reason to use this particular choice is that it is studied only the behavior of solution rather than to make any precise predictions about the neutrino oscillation probabilities. 


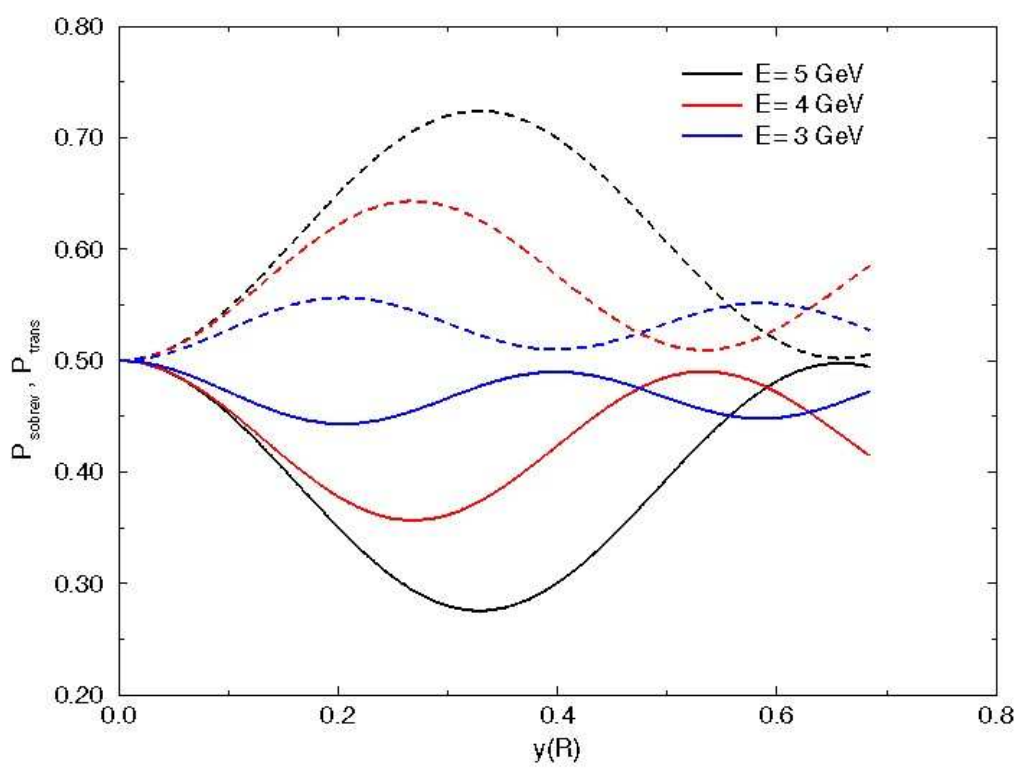

Figure 2: Transition probability (dashed line) and survival probability (solid line) considering two neutrino flavors for 3, 4 and 5 GeV energy values.

To observe the energy influence in the oscillation amplitudes, in figure 2 it is shown the oscillation probabilities of neutrinos in the framework of two neutrino oscillations that cross the Earth with 3, 4 and $5 \mathrm{GeV}$ energy values and reach it with $\theta=70^{\circ}$ and in the initial state

$$
v_{f}\left(x_{I}\right)=\left(\begin{array}{l}
1 / \sqrt{2} \\
1 / \sqrt{2}
\end{array}\right) .
$$

In the graph, $y$ is the ratio between the distance traveled by the neutrino inside of the Earth and the Earth radius.

In this analysis, it was noted that the first order term of the evolution operator for the variable matter density is negligible since for path elements sufficiently small, the electronic density can be considered constant.

\section{Atmospheric muon neutrino flux}

The up and down muon neutrino fluxes without oscillation are simply obtained by the integration of the production rate in the distance $l$ between the source and the detector. For energy of $5 \mathrm{GeV}$ and zenith angle of $70^{\circ}$, it was obtained the muon neutrino flux

$$
\phi_{v_{\mu}}=4,25076 \times 10^{-4}\left(\mathrm{~cm}^{2} . \text { s.sr.GeV }\right)^{-1}
$$

Even considering oscillation among flavors, the muon neutrino flux, without consider the eletronic chanel, is calculated by the relation

$$
\phi_{v_{\mu}}=\int_{l}^{0} P\left(v_{\mu}, v_{\mu}\right) \frac{\partial \phi_{v_{\mu}}}{\partial l} d l
$$

here $\frac{\partial \phi_{\nu_{\mu}}}{\partial l}$ is the neutrino production rate and $P\left(v_{\mu}, v_{\mu}\right)=\left|v_{\mu}(x)\right|^{2}$.

For neutrinos, the atmosphere can be approximated as vacuum, so the flavor state of the 
muon neutrino produced at the distance $l$ of the detector at the sea level is

it follows that

$$
\left(\begin{array}{c}
v_{\mu}(l) \\
v_{x}(l)
\end{array}\right)=\left(\begin{array}{c}
\cos ^{\Delta_{32} l} / 4 E+i \cos 2 \alpha \sin ^{\Delta_{32} l} / 4 E \\
\sin 2 \alpha \sin \Delta_{32} l / 4 E
\end{array}\right)
$$

$$
P\left(v_{\mu}, v_{\mu}\right) \simeq 1-S^{2} 2 \alpha \sin ^{2} \frac{\Delta_{32} l}{4 E},
$$

where $S^{2} 2 \alpha=4 S^{2} \psi C^{2} \phi\left(1-S^{2} \psi C^{2} \phi\right)$.

Using the oscillation parameters from ref. [13] $\Delta_{32}=3 \times 10^{-3} \mathrm{eV}^{2}, \sin ^{2} \psi=0,6$ and $\sin ^{2} \phi=0,01$, we get $S^{2} 2 \alpha=0,96$. In this case, for energy value of $5 \mathrm{GeV}$ and zenith angle of $70^{\circ}$, one finds

$$
\phi_{v_{\mu}}=4,25048 \times 10^{-4}\left(\mathrm{~cm}^{2} \text {.s.sr.GeV }\right)^{-1}
$$

In the case of down neutrino, part of the path is in the atmosphere, for which the oscillation conditions are the same ones appearing for neutrinos up, and part is inside the Earth. Thus, the flavor state for down neutrinos initially of muon flavor is obtained multiplying the state given in Eq. (23) for the evolution operator in the Earth in zero order of approximation, since the term of first order doesn't contribute significantly.

$$
\left(\begin{array}{l}
v_{\mu}(l) \\
v_{x}(l)
\end{array}\right)=\left[\prod_{j}\left(\begin{array}{cc}
c_{j}+i s_{j} \cos 2 \tilde{\alpha} & -i s_{j} \sin 2 \tilde{\alpha} \\
-i s_{j} \sin 2 \tilde{\alpha} & c_{j}-i s_{j} \cos 2 \tilde{\alpha}
\end{array}\right)\right]\left(\begin{array}{c}
\cos ^{\Delta_{32} l} / 4 E \\
\sin 2 \alpha \sin 2 \alpha \sin ^{\Delta_{32}} / 4 \Delta_{3 E}
\end{array}\right)
$$

The survival probability of muon neutrino is $P\left(v_{\mu}, v_{\mu}\right)=\left|v_{\mu}(x)\right|^{2}$. Muon neutrino flux is obtained multiplying the muon neutrino rate by this probability and integrating in $l$, the result is

$$
\phi_{v_{\mu}}=6 \times 10^{-5}\left(\mathrm{~cm}^{2} . \text { s.sr.GeV }\right)^{-1}
$$

for energy value of $5 \mathrm{GeV}$, with zenith angle of $70^{\circ}$ and oscillation parameters from ref. [13].

\section{Discussion}

The up neutrinos fluxes practically doesn't change when taking into account the flavor oscillation possibility. However the down neutrino flux calculated with oscillation corresponds to approximately $15 \%$ of the flux obtained without considering oscillation. Thus the flavor oscillations are revealed as a possible explanation for an eventual up-down asymmetry.

The extension of the calculation accomplished in this work for different values of energy and zenith angles is direct for $\theta \geq 70^{\circ}$ and since the approach of unidimensional showers can be maintained. Note that from our results the up-down asymmetry for neutrino flux can be used as an element to determine the values of physical parameters related to flavor neutrino oscillation. Varying the energy of neutrinos or incident angle, different oscillation probability is obtained and the parameter values can be properly adjusting to reproduce the asymmetry data

The negligible influence of the first order term of the evolution operator in the Earth allows considering the constant electronic density and equal to $\bar{N}_{j}$ for each element of the path. Disregarding of the disturbance, the oscillation in the context of three families described by the evolution operator in the Earth upper mantle can be introduced without much trouble. 
In a general way, the approximation $\tilde{\Delta}_{31} \approx \tilde{\Delta}_{32} \gg \tilde{\Delta}_{21}$ is not reasonable for atmospheric neutrinos that cross the Earth. Without this consideration, the survival and transition probabilities of muon neutrinos in two family approximation are got like that of Bernabéu [13] or Gonzales-Garcia [14]. However, with these expressions the method of Lisi is not directly aplicable. Therefore, it is viable to extend to three neutrino flavors the procedure of Lisi since it could be aplied in all cases. This is the next step of our work.

\section{References}

[1] G. L. Fogli, E. Lisi, A. Marrone, A. Palazzo, A. M. Rotunno. Global analysis of neutrino masses and mixing. Prog. Part. Nuc. Phys. 57 (2006) 61-67.

[2] S. M. Bilenky. Neutrino masses, mixing and oscillations. Prog. Part. Nuc. Phys. 57 (2006) 71.

[3] T.K. Gaisser. Atmospheric neutrino flux: a review of calculations. Nuc. Phys. B 87 (2000) 145.

[4] P. Lipari. The geometry of atmospheric neutrinos production. Astrop. Phys. 14 (2000) 153.

[5] E. Lisi, D. Montanino. Earth Regeneration in solar neutrino oscillations: an analytic approach. Phys. Rev. D 56 (1997) 3.

[6] P. Lipari. Lepton spectra in the earth's atmosphere. Astrop. Phys. 1 (1993) 195.

[7] L. V. Volkova, G. T. Zatsepin, L. A. Kuz'michev. The cosmic-ray muon spectrum at sea level and the primary cosmic-ray nucleon spectrum. Sov. J. Nucl. Phys. 29 (1979) 645.

[8] L. V. Volkova. Energy spectra and angular distributions of atmospheric neutrinos. Sov. J. Nucl. Phys. 31 (1980) 784.

[9] W. Lohmann, R. Kopp, R. Voss. Energy loss of muons in the energy range 1-10000 GeV. CERN Y. Rep. 85-03 (1985) 29.

[10] T. K. Gaisser. Cosmic Rays and Particle Physics. Cambridge University Press (1990).

[11] T. K. Kuo, J. Pantaleone. Neutrino oscillations in matter. Rev. Mod. Phys. 61 (1989) 937.

[12] L. Wolfenstein. Neutrino oscillations in matter. Phys. Rev. D 17 (1977) 2369.

[13] J. Bernabéu, S. Palomarez-Ruiz, S. T. Petcov. Atmospheric neutrinos oscillations, $\theta_{13}$ and neutrino mass hierarchy. Nuc. Phys. B 669 (2003) 255-276.

[14] M. I. Gonzales-Garcia, C. Peña-Garay. Neutrino masses and mixing one decade from now. Nuc. Inst. Met. Phys. Res. A 451 (2000).

[15] A. M. Dziewonski, D. L. Anderson. Preliminary Earth reference model. Phys. Earth Planet. Inter. 25 (1981) 297. 